\title{
Physician Knowledge, Financial Incentives and Treatment Decisions for Depression
}

\author{
Roland Sturm* and Kenneth B. Wells \\ RAND, Santa Monica, CA, USA
}

\begin{abstract}
Background: Two important policy levers to affect health care delivery are financing and informational interventions. Unfortunately, these two approaches have not been considered simultaneously and little is known about how their effects compare.

Aims of the Study: This paper estimates the relative role of financial incentives (prepaid versus fee for service) and provider information (perceived knowledge of antidepressant medications and skill in counseling for depression) on quality of care for less and more severely depressed patients and their health and cost outcomes.

Methods: We develop a theoretical model of provider behavior and estimate a reduced form using a multinomial probit model with heteroskedastic covariances. The likely effects of changing provider knowledge about depression treatment in primary care are then simulated and contrasted with the effects of a shift toward prepaid managed care as opposed to fee-for-service care. The empirical model is estimated using data from the Medical Outcomes Study.

Results: We conclude that financing and information have different effects and that their combination can achieve the conflicting goals of improved health outcomes and reduced direct treatment goals. Moreover, including family income as one important dimension of social cost suggests that the combination of informational interventions and a shift to prepaid care may dominate either one intervention in isolation from a social cost perspective. Specifically regarding information, we found that increasing provider knowledge could have the highly desirable effect of greater targeting of treatments to sicker patients while not raising overall treatment rates much - a treatment pattern that many hoped managed care could achieve, but for which there has been little evidence.

Conclusions: Our analysis illustrates the value of considering these widely different policy goals simultaneously. We learned that variation in physician knowledge generally had stronger associations with clinically relevant practice patterns for depression than did a complete change in financing strategy. The moderate change in perceived knowledge we simulated (not near the extremes of observed values of perceived knowledge) was associated with enough improvement in appropriateness of care to more than offset the reduction in appropriateness with a complete shift from feefor-service to prepaid managed care.

Implications for Health Policy: The paper demonstrates the importance of considering different interventions simultaneously.
\end{abstract}

* Correspondence to: Roland Sturm, RAND, 1700 Main Street, Santa Monica, CA 90401, USA. Tel: (310) 393-0411 ext. 6164. E-mail: Roland Sturm@rand.org

Contract grant sponsor: National Institute of Mental Health

Contract grant number: MH 54147 (Sturm), MH 54623 (Wells)

Contract grant sponsor: AHCPR

Contract grant number: HS08349
Combining informational and financial interventions simultaneously can achieve better quality of care and reduce health care costs, something neither intervention can in isolation. (C) 1998 John Wiley \& Sons, Ltd.

Received 24 April 1997; accepted 28 April 1998.

\section{Introduction}

Depression is a highly prevalent condition that is associated with substantial morbidity and social costs. ${ }^{1-4}$ Although several treatments, including antidepressant medication and specific psychotherapies, are of demonstrated efficacy in depression, ${ }^{5,6}$ most depressed persons receive either no care or no specific appropriate treatment for depression, even when seeking care. ${ }^{2}$ Yet while many seriously depressed patients go without appropriate treatment, many others receive treatment that provides little benefit for them. This paper develops an empirical model to study the role of information and financial incentives in addressing this inefficiency.

Economists typically consider the simultaneous problems of under-and overuse of care to be functions of incentives on the supply or demand side: overuse as a consequence of moral hazard on the demand side when insured patients pay less than the marginal cost of treatment; underuse as a consequence of supply-side cost sharing when providers act too little as agents for the patients. In this framework, supply-side cost sharing incentives, such as capitated payments to providers, are thought to be efficient tools that primarily reduce questionable care and target health care dollars to patients most likely to benefit from treatment.

In contrast, most clinical and health services research pays little attention to incentives and primarily attributes low quality or inappropriate care to physician ignorance or bias about diagnosing and treating depression. Consequently, interventions tend to focus on information, ${ }^{8-11}$ although the most successful studies also alter delivery characteristics. ${ }^{12,13}$ Provider knowledge may be important as even a relatively weak educational program in Sweden is claimed to have resulted in improved health outcomes and overall social savings to society. ${ }^{14}$

Unfortunately, these two approaches-informational and financial incentives-have not been considered simultaneously and little is known about how their effects 
compare. This paper estimates the relative role of financial incentives (prepaid versus fee for service) and provider information (perceived knowledge of antidepressant medications and skill in counseling for depression) in accomplishing two goals: increasing use of effective treatments for depression overall-an important clinical care goal given the large gap between current practice and practice guidelines recommendations-and improving the targeting of such treatments to the sickest patients, an important goal for plans to increase value of care. Regarding the first goal, several studies found that quality of care for depression is particularly poor in primary care settings, the sector that treats more than half of all depressed persons receiving mental health care, ${ }^{2,15}$ and we therefore focus on this sector. Regarding the second goal, some studies found that outcomes for depression were worse in prepaid than in fee-for-service settings and those effects were concentrated among the sickest patients, ${ }^{16,17}$ suggesting that we need to study the effects of information and financing by patient severity. Our main research question is the following: what do financial incentives (as represented by fee for service versus prepaid) or provider knowledge achieve in terms of these goals?

\section{Methods}

\section{Theoretical Model}

Our behavioral economic model assumes that providers choose a treatment modality for each patient based on the providers' expectation of benefits to the patient (which depends on patient characteristics, such as age, diagnosis and severity) and marginal costs to the providers' practice for each treatment modality (which depends on the financing system). Treatment benefits differ across patients according to their health status, but the provider does not know the true benefit that a specific treatment will provide to a particular patient before initiating treatment. The quality of the prognosis depends on the provider's knowledge and understanding of the course of the illness, patient sickness and the effects of treatment. How the provider values patient benefits and costs for each potential treatment is an unobserved latent variable, which we call the provider's 'utility' of the treatment, and the provider chooses the treatment with the highest expected value. The latent variable is parametrized as a function of provider information, financing and patient characteristics to measure the influence of these factors.

The standard modeling approach in economics is that health care providers are agents of their patients and their practice/employers and are concerned about both patient benefits from treatment $(\mathbf{B})$ and costs to their practice $(\mathbf{C})$. In this paper, we consider the choice between several discrete types of treatment, including no treatment, rather than the quantity of a homogeneous service as in most economic research. The cost argument of the provider's utility function is the net utility of providing a treatment in terms of effort and income, differing across treatments and financial arrangements.

Providing treatment $j$ costs the provider a net amount $C_{j}$, which is equal to zero for no treatment. If $C_{j}$ is zero for all treatments, clinicians act as perfect agents for patients (under incomplete information this is not the same as making the optimal choices for patients). $C_{j}$ is positive if the reimbursement for treatment $j$ is less than the provider's marginal costs (supply-side cost sharing, a typical feature of prepaid arrangements) and negative if the reimbursement is higher than the marginal costs.

Treatment benefits differ across patients according to their health status, copayments, and observable and unobservable individual characteristics. The physician values patient $i$ 's (ex post) benefit of treatment $j$ as $B_{i j}$ (with no treatment having benefits of zero as a normalization). The value of $B_{i j}$ is low or negative for patients who benefit little from treatment $j$ because it is inappropriate for their health condition, too costly for the patient or because it does not work. The provider does not know the true benefit that treatment will provide to a particular patient before initiating treatment. The quality of the prognosis depends on the provider's knowledge and understanding of the course of the illness, patient sickness, the effects of treatment, and patient preferences. The provider's expected value of patient benefit is $B_{i j}=B_{i j}+\mu_{i j}$, or written as a vector for all treatments

$$
\hat{B_{i}}=B_{i}+\mu_{i}
$$

where $\mu_{i}$ is a random draw from the provider's subjective information probability density function $g(\boldsymbol{\mu})$, independent from the patient's true benefits. A provider for whom $\mid \mathbf{B}-$ $\mathbf{B} \mid$ is large is poorly informed about the patient's outcome and preferences and a provider for whom $|\mathbf{B}-\mathbf{B}|$ is very small is well informed. The discrepancy between expected and actual benefits could be caused by systematic biases $\left(E\left(B_{i}\right) \neq B_{i}\right.$ or $\left.E(\mu) \neq 0\right)$ or by the dispersion of $\mu$. If $E(\mu)=0$ and $V(\mu)>0$, clinicians do not systematically misestimate benefits, although they also never estimate the benefit exactly right for any patient. The mean squared error $E\left(\mu \mu^{\prime}\right)$ captures both bias and dispersion and a natural measure of information is therefore $I=E\left(\mu \mu^{\prime}\right)^{-1}$. This linear utility function and expected benefits representation lends itself best to an empirical model, although it is not completely general. Similar models of uncertainty when $\mu$ was a scalar have been used in the consumer choice literature to describe unproductive consumption ${ }^{18}$ and insurance purchases. $^{19}$

To understand the implications of this model, first reconsider the single choice consumer example of Gertler, Sturm and Davidson ${ }^{19}$ in a health care context. Assume that the health care provider can only choose between one specific treatment and no treatment for a patient and that no treatment has zero costs and benefits. With complete information about outcomes, a physician provides the treatment if $B_{i}>C$. The probability of treatment is $Y=\int_{C}^{\infty} f(t) \mathrm{d} t$, where $f(B)$ is the density distribution of treatment benefits in the provider's patient population. In Figure 1, $Y$ is the area to the right of $C$. Under incomplete 


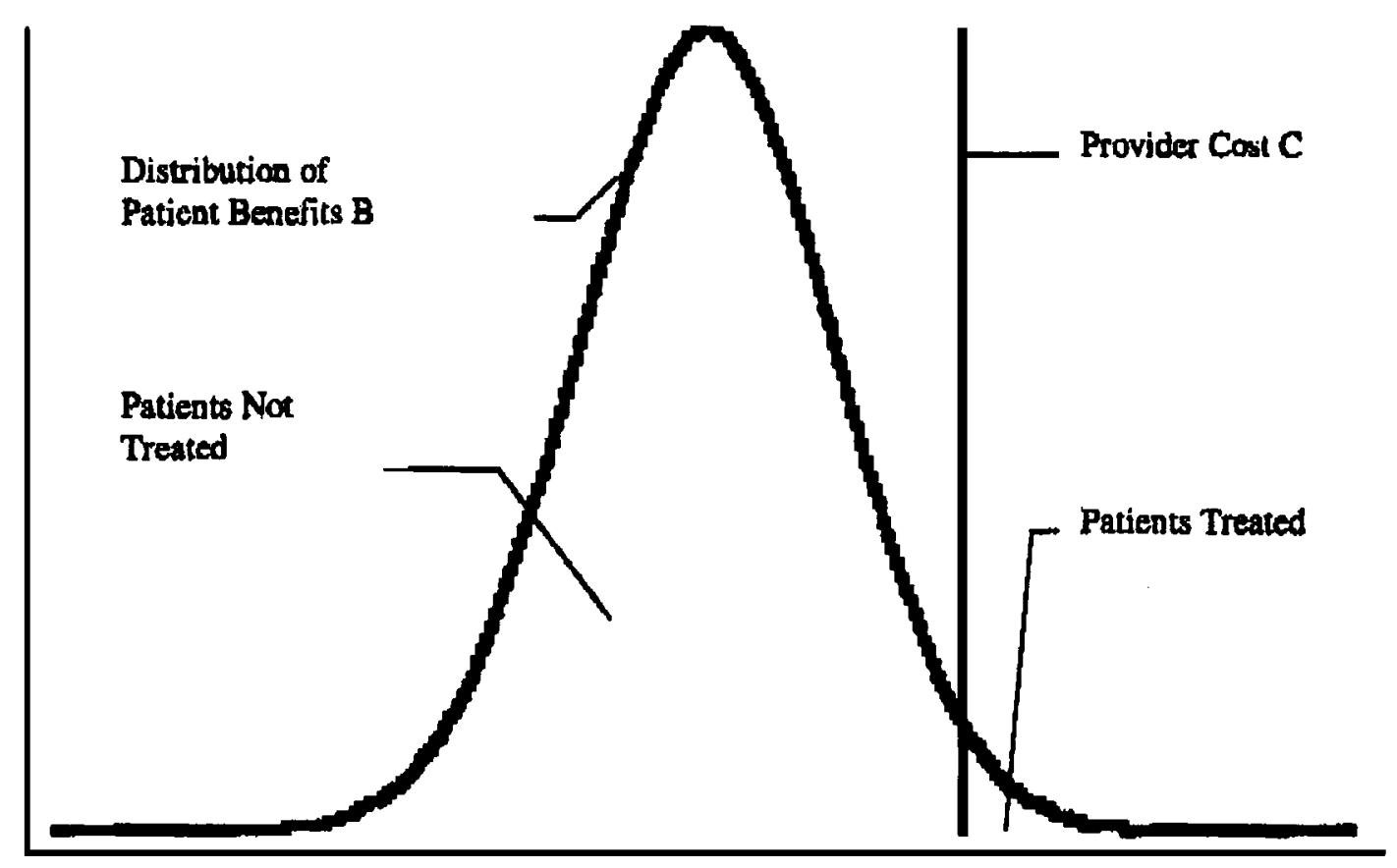

Figure 1. Patients are treated if expected benefits exceed costs

information, clinicians provide the treatment only if $\hat{B}_{i}>$ $C$ and the probability of treatment is $Y=\int_{C}^{\infty} h(t) \mathrm{d} t$, where $h(B)$ is the density function of the expected value $B$.

While systematically over- or underestimating benefits always biases the probability of treatment in the same direction, the effect of an increase in dispersion depends on whether provider costs cut the true benefit distribution to the left or the right of the mode. Figure 2 shows the distribution of true benefits $f(B)$ and the distribution of perceived benefits $h(B)$ under the assumption that the mean of $\mu$ is zero. In Figure 2(a), $C$ is to the left of the mode and increased dispersion shifts mass to the left of $C$, which means that the aggregate number of treatments decreases. In Figure 2(b), $C$ cuts the distribution to the right of the mode and a mean-preserving increase in dispersion shifts mass to the right of $C$, leading to increased treatment. Thus, the probability of choosing treatment changes with the amount of information even if the physician is completely unbiased about the expected benefits.

Capturing both the mean and variance effect of the information distribution is important in an empirical model as these two structural explanations lead to different policy recommendations. If providers systematically underestimate treatment benefits to patients $(E(\mu)<0, V(\mu)=0)$, providing better reimbursement for those treatments can offset this bias to provide the desired treatment probabilities. In mental health, low rates of appropriate care for clinically depressed patients by primary care physicians is often considered to be evidence for such systematic biases or 'mental health stigma' among poorly informed physicians. Given the many unsuccessful attempts to improve physician decisions through informational interventions, such as outcome feedback, changing incentives may be an efficient approach to achieve desired treatment rates. Of course, low treatment rates may

TREATMENT DECISIONS FOR DEPRESSION simply be a consequence of provider incentives which lead to undertreatment (compared to optimal care from the patient perspective) if $C>0$, in which case the failure of informational interventions is hardly surprising. On the other hand, if the reason for undertreating sick patients has been an inability among providers to appropriately target care $(E(\mu)=0, V(\mu)>0)$, increased reimbursement is inefficient as its main effect is to increase the proportion of inappropriately overtreated patients.

\section{Statistical Model}

In the general case, the physician provides treatment $j$ if it has the highest perceived net utility among all choices, i.e., $U_{j}=B_{j}-C_{j} \geqslant U_{k}=B_{k}-C_{k}, \forall k \neq j$. In this application to depression, we consider four choices (counseling only, antidepressant medication, minor tranquilizers only or none of the three). However, we describe the econometric model generally, so that it can be immediately applied to other settings with more or fewer than four choices and other covariance specifications than we used. To write the probability of choosing a treatment compactly, define

$$
A_{j}=\left[\begin{array}{ccccccc}
1 & & & & -1 & & \\
& 1 & & & -1 & & \\
& & \ddots & & \vdots & & \\
& & & 1 & -1 & & \\
& & & & -1 & 1 & \\
& & & & \vdots & \ddots & \\
& & & & -1 & & 1
\end{array}\right]
$$

and

$$
U_{-j}=A_{j} U=\left(U_{1}-U_{j}, U_{2}-U_{j}, \ldots,\right.
$$



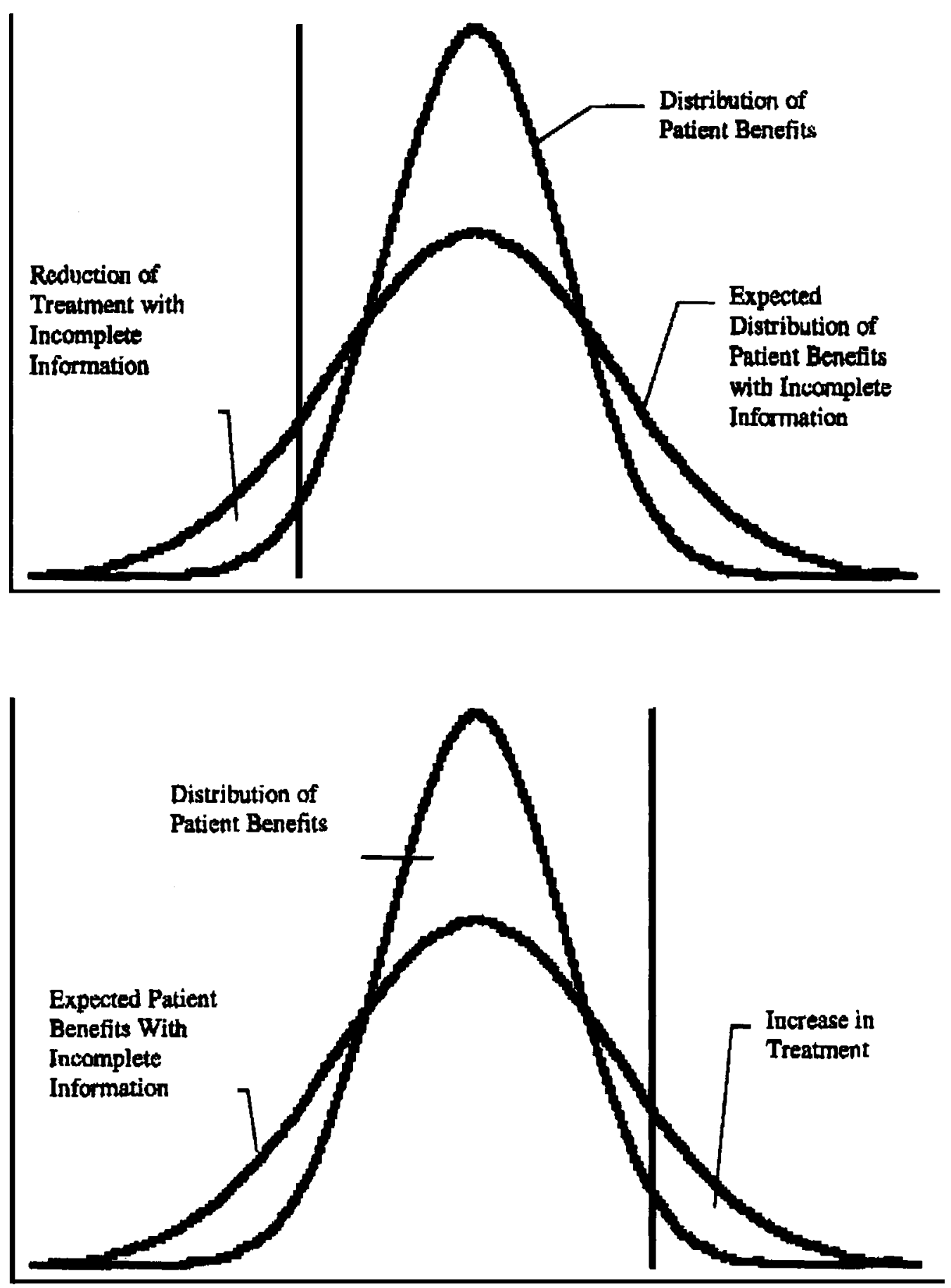

Figure 2. (a) Less knowledgeable providers undertreat when most patients would benefit from treatment. (b) Less knowledgeable providers overtreat when few patients would benefit from treatment

$$
\left.U_{j-1}-U_{j}, U_{j+1}-U_{j}, \ldots, U_{j}-U_{j}\right)
$$

then the probability that treatment $j$ is provided is

$$
P_{j}=P\left(T_{j}=1\right)=\int_{-\infty}^{\infty} I\left(U_{-j} \leqslant 0\right) f\left(U_{-j}\right) \mathrm{d} U_{-j}
$$

where $I(Q)$ is the indicator of the event $Q$ and $f$ is the density function of $U_{-j}$.

For the conditional utility function, we consider a linear function:

92

(C) 1998 John Wiley \& Sons, Ltd.

$$
U_{i j}=\hat{B}_{i j}-\alpha C_{j}
$$

A linear utility function imposes a constant marginal rate of substitution between patient benefits and costs, ${ }^{19}$ although this could easily be generalized by having $\alpha$ depend on patient benefits or costs. Because no direct measures of the utility are available, $B_{i j}$ and $C_{j}$ need to be parametrized as functions of observed patient and provider individual characteristics. Because the Medical Outcomes Study does not have direct measures of marginal costs, this distinction

R. STURM AND K.B. WELLS 
is somewhat irrelevant in the depression application, but it will become important in data that directly measure costs and provide a tighter fit between theory and data. The provider's expected utility from treatment $j$ for patient $i$ is

$$
U_{i j}=\hat{B}_{i j}-C_{j}=\beta_{0 j}+\sum_{k=1}^{k} \beta_{k j} X_{i k}-\sum_{l=1}^{L} \gamma_{l} Y_{l}+\epsilon_{i j}+\mu_{i j}
$$

where $\mathbf{X}$ is a vector of characteristics that affect patients' benefits, including sociodemographic variables, insurance status, and especially detailed measures about mental health status, and $\mathbf{Y}$ are characteristics that affect the provider's cost, such as system of care and reimbursement characteristics. The alternative specific intercept picks up the overall mean in the utility of expected benefits, and is augmented by observed and unobserved individual characteristics. The coefficients on the individual characteristics $(\mathbf{X}$ and $\mathbf{Y})$ are allowed to differ by alternative. Poor mental health and a clinical depressive disorder, for example, will make antidepressant medication more more valuable than no treatment or minor tranquilizer use, whereas a capitation contract (fixed payments per enrolled individual regardless of service use) for office visits, but not medication costs, will make counseling more expensive than medication treatment to the provider.

Because the choice depends only on the difference between two utilities and not on their absolute value, only differences can be identified. It is equivalent whether one normalizes the utility of no specific treatment to zero (the assumption in the theoretical model) or whether one considers the difference between each treatment (counseling, antidepressant medications and minor tranquilizers) and no treatment. In this case, the intercept and the coefficients on the $\boldsymbol{X}$ are interpreted as affecting the marginal utility of the expected benefits from providing a specific treatment for depression.

The random components in (6) consist of two (uncorrelated) additive factors; neither one is observed by the econometrician. The first random term, $\epsilon_{i j}$, reflects 'true' factors about patient benefits and costs observed by the clinician, but not by the econometrician. The second term $\left(\mu_{i j}\right)$ measures the divergence between expected and true benefits. The distribution of $\mu_{i j}$ determines how incomplete information affects the distribution of treatment and this term would be zero if the clinician had perfect information about the patient's benefits. We assume that $\boldsymbol{\epsilon}_{i} \sim N(0, \boldsymbol{\Omega})$, where $\boldsymbol{\Omega}$ is a $J \times J$ matrix ( $J=4$ choices in this paper), and that the conditional subjective probability distribution is $\boldsymbol{\mu}_{i} \mid \mathbf{I} \sim N(E \mu(\mathbf{I}), \mathbf{\Sigma}(\mathbf{I}))$, where $\mathbf{I}$ is a vector of measures of the provider's self-rated understanding of medication and counseling treatment for depression. This representation assumes that both random effects are independent across patients, which does not seem problematic for a crosssection of patients treated by different providers. However, it would not be appropriate when many patients are sampled from a small number of providers if a clinician repeatedly misjudges benefits in the same way, as this introduces a correlation between the vectors $\boldsymbol{\mu}_{i}$.
Compare this model with the most common empirical estimator in qualitative choice models, the multinominal logit model (MNL). The MNL can be written as in equation (6), but without the second term $\left(\mu_{i j}\right)$ that measures the divergence between expected and true benefits. In the MNL, the $\boldsymbol{\epsilon}_{i}$ are independently distributed with an extreme value (Gumbel) distribution; in the multinomial probit (MNP), the $\boldsymbol{\epsilon}_{i}$ are jointly normal. The special case of the multinomial probit in which there is no second term and the $\boldsymbol{\epsilon}_{i}$ are independently distributed and have identical variance has therefore the same basic structure as the MNL.

Not all of the variance parameters can be identified and we impose two standard normalizations on the variancecovariance matrix..$^{20,21}$ First, because choice probabilities depend only on differences in utility and therefore on the distribution of differences in the error, the dimensionality of $\boldsymbol{\Omega}$ can be reduced by unity, which implicitly normalizes one choice specific random component to be zero (here no treatment). A second normalization is necessary because the probability $\Phi(V, \boldsymbol{\Omega})$ is the same as the probability $\Phi(k V$, $\left.k^{2} \boldsymbol{\Omega}\right)$. Additional variance effects that differ across individuals and choices according to other covariates can be added without an identification problem and to estimate the effect of information on the dispersion of the value of expected benefits, we add the covariance matrix $\mathbf{\Sigma}(\mathbf{I})$.

The theoretical advantage of the multinomial probit over other functional specifications is that it allows correlations among the $\boldsymbol{\epsilon}$, which could overcome the sometimes unrealistic 'independence-of-irrelevant-alternatives' constraint. However, this theoretical advantage may not be as useful practically. When adding additional covariance parameters for $\boldsymbol{\Omega}$, the models failed to converge because the Hessian became negative during iterations. One can constrain the Hessian to be positive with a Cholesky decomposition, but this requires an unconstrained covariance matrix and the large number of additional covariance parameters was probably the reason that the model did not converge in the unconstrained covariance case either. This seems to be a general difficulty with multinomial probit models as Keane ${ }^{22}$ first pointed out. We encountered similar difficulties in the paper by Gertler et al. ${ }^{19} \mathrm{We}$ eventually used an identity matrix for $\boldsymbol{\Omega}$.

Under these assumptions, $U_{-j}$ is distributed normally with mean $A_{j} U$ and variance $A_{j}(\boldsymbol{\Omega}+\mathbf{\Sigma}(\mathbf{I})) A_{j}^{\prime}$ and $P_{j}=P\left(T_{j}=1\right)$ $=\int_{\infty}^{0} \phi_{A_{j} U, A_{j}(\Omega+\Sigma) A_{t}^{\prime}}(t) \mathrm{d} t$. The likelihood function for $N$ patients is then

$$
L=\prod_{i=1}^{N} \prod_{j=1}^{J} P_{j}^{I\left(T_{j}=1\right)}
$$

which is a multinomial probit model with a heteroskedastic covariance matrix as a function of provider knowledge.

When constraining the variance parameters to be identical across treatment modalities and patients, the qualitative results of this model are necessarily identical to the multinomial logit model, which we used for preliminary specification tests. In particular, we tested whether other provider characteristics (such as provider age, gender or ethnicity or delivery characteristics, such as distinguishing 
group practices from staff-model HMOs) affected treatment decisions, but found no significant or noteworthy effects and therefore omitted those variables from the final model. We distinguished family practitioners from other general medical providers (primarily internal medicine) because the perceived counseling skills and medication knowledge are significantly higher among family practitioners than among other primary care providers - but these differences are not associated with treatment rate differences. We also tested whether patient factors other than illness affected treatment decisions and found that patient age and ethnicity were highly significant, but gender, education and marital status were not and we excluded the latter three variables. We also tested for an interaction between payment system type and perceived knowledge, since the knowledge effect on process of care could differ if providers are more behaviorally constrained under prepaid care, but found no evidence for it.

\section{Data}

The Medical Outcomes Study (MOS) is an observational study of adults in alternative systems of care in three urban areas (Boston, Chicago and Los Angeles). In each geographic area, clinicians in Health Maintenance Organizations (HMOs), large multispecialty group practices and solo/small group practices were enrolled in the study, including 14 large practices (some of them with several locations) with prepaid care. During the initial cross-sectional data collection (1986-1987), patients of the enrolled providers were screened for depressive symptoms, using a brief self-administered instrument, followed by a telephone interview that included the depression section of the NIMH Diagnostic Interview Schedule. The telephone interview was limited to respondents who had an ongoing relationship with an MOS clinician, could complete self-administered questionnaires and did not have competing acute physical conditions that severely limited functioning. Patients in the longitudinal portion ranged from patients with current major depressive and dysthymic disorder to those with some symptoms, but no current disorder, which we refer to as subthreshold or minor depression. Patients with lifetime mania were excluded. The MOS design has been described more fully before. ${ }^{2,23}$ Details of measures can be found in the book by Wells et al. ${ }^{2}$

Data on care for the acute episode at baseline are available for 531 primary care patients. We defined four mutually exclusive categories of care: (i) counseling for depression only (patient receives counseling but not psychotropic medication); (ii) any effective antidepressant medication (patient may or may not receive minor tranquilizers or counseling); (iii) minor tranquilizers as the only psychotropic medication (with or without counseling for depression); (iv) none of these treatments. We classified each patient into one of these four groups. About $20 \%$ received only counseling (i), $6 \%$ any effective antidepressants (ii), $14 \%$ minor tranquilizers as the only psychotropic medication (iii) and the remainder none of these (iv). Defining these mutually exclusive categories clarifies the large role of no treatment, which was obscured in our earlier work that considered counseling, antidepressants, and minor tranquilizers individually. ${ }^{24}$

Data on use of psychotropic medications were obtained from patient interviews conducted by study research clinicians. The medication data include the name of each drug used in the prior month or regularly in the prior six months and the daily dosage. We distinguished any minor tranquilizer or sedative/hypnotic from antidepressant medication (heterocyclic, monoamine oxidase inhibitor or newer antidepressant) because only the latter are known to be efficacious for depression. Antidepressant dosages were categorized as within a therapeutic range or below the minimum therapeutic dosage. ${ }^{15,25}$ An operational definition of effective psychotherapy in typical practice settings is difficult and we used an indicator of at least minimal 'counseling' ( 3 min or more), based on clinician-reported data after the index visit in which depression was identified. This is an indicator of a course of treatment because it predicted an additional 11 mental health visits. ${ }^{24}$

The panel included patients with no current or lifetime depressive disorder $(42 \%)$, patients with lifetime but not current disorder (13\%), current major depressive disorder (19\%), current dysthymic disorder (12\%) and concurrent major and dysthymic disorder (double depression, 14\%). Because treatment likelihood should depend on patient illness, the analysis controls for each patient's initial psychological health status, which is a global measure that summarizes multiple health status measures, ${ }^{17}$ including diagnostic status and severity of symptoms and patient demographics. Provider information is self-rated and distinguishes knowledge of antidepressant medication (mean 60 on a $0-100$ scale, standard deviation 23) and perceived skill in counseling for depression (mean 58 on a 0-100 scale, SD 24), both of which are single-item measures, asking the physician to rate their knowledge about this particular treatment. Although no further psychometric evaluation of those measures (such as retest reliability) is available, the predictive power of those measures was studied. Physicians of depressed patients who received any psychotropic medication or counseling, compared to those receiving neither treatment, have significantly higher perceived knowledge about antidepressant medications (mean 63 versus 57, $p=$ 0.01 ) and counseling skill (mean 64 versus 54, $p<0.01$ ). Moreover, providers with higher perceived medication knowledge at baseline were significantly more likely to maintain their patients with recurrent depression (current disorder and three or more prior episodes) on antidepressant medication over time ( $p<0.05$ after one year, $p<0.10$ after two years with lower significance due to the smaller sample size caused by attrition). This baseline perceived knowledge predicted subsequence clinical competence (based on independent assessments of treatments received), supporting the validity of this measure.

We used the final model to study the effects of payment type and clinician perceived knowledge, i.e. we specified patient characteristics (for example, 40-year-old white female with double depression) and compared the probability of care under various scenarios of provider knowledge and 
payment. We illustrate the effects on targeting by contrasting results for patients with double (severe) and subthreshold (minor) depression. The predictions are standardized to the sociodemographic characteristics of the MOS primary care depression panel. Rather than simulate extreme knowledge changes that might be unrealistic to achieve, we defined a moderately strong effect size, equivalent in terms of standardized scale points to change in diagnostic accuracy observed in an educational intervention study, ${ }^{11}$ which found that a $3 \frac{1}{2} \mathrm{~h}$ seminar improved the diagnostic accuracy for dysthymia and major depression significantly relative to control clinicians. On a standardized 0-100 scale (centered at the average), the improvement in diagnostic accuracy was about $30-40$ points, and we simulate a 40 -point increase in standardized versions of our knowledge/perceived skill scales, centered at the average. This approach could understate the potential of more intensive informational interventions compared to a complete switch from fee-forservice to prepaid care, but we note that this is four times the difference in knowledge in the MOS between clinicians who deliver any as opposed to no treatment to their depressed patients (i.e., it does not seem to be a small effect). For purposes of guiding policy formulation at the early stage of choosing a target goal, the precision of the prediction parameters often matters less than the direction of findings; modeling smaller changes results in the same relative relationships but at less extreme absolute levels of impact.

While changes in quality of care (i.e., rates of use of treatments of known efficacy) are interesting in their own right, policy makers are most interested in measures of costs, particularly direct treatment costs and morbidity outcomes, as proxies for indirect social costs. While we can estimate the change in quality of care measured by processes of care as a function of information and financing, our simulation of the cost and morbidity has to rely on cruder assumptions. For that second link, we use previously published estimates of process-outcome relationships for severely depressed patients in all sectors (including mental health specialty). Our cost estimates are derived from the finding that effective antidepressant medication is associated with an additional five and counseling with an additional 11 visits for mental health care (controlling for severity and provider specialty). ${ }^{24}$ Our conclusions could underestimate the cost savings resulting from a shift to prepaid care for primary care patients, but not by much as the main cost differences by payment are among specialty patients, not primary care patients. ${ }^{26}$ We may also overstate the cost implications for subthreshold patients. Regarding morbidity outcomes (reductions in serious functioning limitations), we apply the average estimated effect of counseling and effective antidepressant use (derived from the most severely depressed patients) to all patients with depressive disorder and assume there was no detectable effect of treatments for subthreshold patients, the pattern observed in the MOS data base. ${ }^{24}$ If depression treatments improve functioning among subthreshold patients, we could underestimate the changes in health improvements with changes in treatment for this group.

\section{Results}

Table 1 shows the distribution of treatment for 100 (actual) primary care depressed patients under care as usual under the distribution of financing and knowledge in the participating practices. These are percentages and standard deviations are therefore omitted. Patients with minor depression (i.e. no clinical disorder) are the most likely to receive no treatment or counseling, while patients with severe depression are more likely to receive psychotropic medication; yet even 47 of 100 severely depressed patients are currently untreated. Table 2 provides the descriptive statistics for the regressor variables in the final model.

Table 3 provides the estimated coefficients of the multinomial probit model, which used counseling skill, medication knowledge (knowmed) and age divided by 100 . The first set of regression coefficients refer to counseling only, the second to antidepressants, the third to minor tranquilizers; no treatment is the baseline category. The last two coefficients in Table 3 measure the effect of knowledge on the covariance matrix. The specification for each covariance term was $(100-$ knowledge $) / 100$ times the coefficient. Therefore, increased antidepressant medication knowledge decreases the variance associated with the provider's utility of providing antidepressants (the estimated coefficient is 3.21), increased counseling skills decreases the variance associated with the utility of counseling (coefficient 4.86). The variance of $\mu_{i j}$ for a less knowledgeable physician (when $j=$ antidepressants or counseling) is therefore relative large compared to the variance of the 'random' error vector $\boldsymbol{\epsilon}_{i}$ (equal to unity in the estimation reported in Table $\mathbf{3}$ ).

We tested this specification against several alternatives using likelihood ratio tests. The variance effects of knowledge (the last two coefficients in Table 3) are jointly highly significant $\left(\chi^{2}=10.99\right.$, two degrees of freedom, $\left.p<0.01\right)$, thus improved knowledge is associated with improved targeting of treatment to sicker patients. There is no significant preference (or aversion) for antidepressant medication, counseling or minor tranquilizer over no treatment as a function of medication knowledge, i.e. increased medication knowledge does not significantly shift the mean utility of treatments. However, increased counseling skills are significantly associated with a tendency to prescribe minor tranquilizers and this effect was strong enough in a joint test of whether there is any effect of knowledge on mean utilities $\left(\chi^{2}=31.8\right.$, six degrees of freedom, $\left.p<0.01\right)$. We therefore maintained the knowledge variables in the mean utility specification. Financing affects the mean utility of treatment $\left(\chi^{2}=12.4\right.$, three degrees of freedom, $p<$ 0.01 ) by shifting treatment away from counseling and towards medication.

Because regression coefficients are difficult to interpret, Table 4 simulates changes in distribution of treatment with a payment switch (a complete switch from fee-for-service to prepaid care) or a knowledge increase (a 40-point change), or both, using the estimated model of Table 3. The last column of Table 4 summarizes the overall change in 
Table 1 . Treatment rates per 100 primary care depressed patients

\begin{tabular}{|c|c|c|c|c|}
\hline Type of depression & Counseling & Antidepressants & $\begin{array}{l}\text { Minor } \\
\text { tranquilizer }\end{array}$ & No treatmen \\
\hline Subthreshold (not clinically depressed) & 21 & 2 & 12 & 65 \\
\hline Double depression (two clinical disorders simultaneously) & 23 & 13 & 17 & 47 \\
\hline
\end{tabular}

Table 2. Descriptive statistics $(n=531)$

\begin{tabular}{lcrrr}
\hline Variable & Mean & Std. Dev. & Min & Max \\
\hline Medication knowledge & 60.1 & 23.0 & 0 & 100 \\
Counseling skill & 57.9 & 23.6 & 0 & 100 \\
Prepaid (1 = yes) & 0.45 & 0.50 & 0 & 1 \\
Family practice $(1=$ yes) & 0.31 & 0.46 & 0 & 1 \\
Patient psychological sickness (higher is worse) & 0.12 & 0.93 & 0 \\
Patient current depressive disorder (1 = yes) & 0.45 & 0.50 & 18.1 & 0 \\
Patient age (years) & 47.3 & 16.1 & 86.1 \\
Patient ethnicity $(0=$ white, $1=$ others) & 0.27 & 0.44 & 1 \\
\hline
\end{tabular}

Table 3. MNP maximum likelihood estimates

\begin{tabular}{|c|c|c|c|c|}
\hline Variable & Coefficient & Std. err. & Est./SE & Prob. \\
\hline \multicolumn{5}{|l|}{ Counseling } \\
\hline Constant & -5.28 & 4.99 & -1.32 & 0.09 \\
\hline Counseling skill & 4.53 & 3.68 & 1.23 & 0.11 \\
\hline Medication knowledge & 1.30 & 1.44 & 0.90 & 0.18 \\
\hline Psychological sickness & 0.42 & 0.23 & 1.84 & 0.03 \\
\hline Prepaid & -1.31 & 0.96 & -1.37 & 0.09 \\
\hline Age & -0.31 & 1.44 & -0.22 & 0.41 \\
\hline Ethnicity & -1.46 & 0.99 & -1.48 & 0.07 \\
\hline Family practice & 0.06 & 0.41 & 0.15 & 0.55 \\
\hline \multicolumn{5}{|l|}{ Antidepressant medication } \\
\hline Constant & -8.17 & 3.69 & -2.22 & 0.01 \\
\hline Counseling skill & 0.067 & 1.18 & 0.06 & 0.48 \\
\hline Medication knowledge & 3.86 & 2.58 & 1.50 & 0.07 \\
\hline Psychological sickness & 2.05 & 0.63 & 3.28 & $<0.001$ \\
\hline Prepaid & 0.48 & 0.54 & 0.89 & 0.19 \\
\hline Age & 4.16 & 2.21 & 1.88 & 0.03 \\
\hline Ethnicity & -2.09 & 0.91 & -2.3 & 0.01 \\
\hline Family practice & -0.50 & 0.51 & -0.96 & 0.17 \\
\hline \multicolumn{5}{|l|}{ Minor tranquilizer } \\
\hline Constant & -3.67 & 0.55 & -6.7 & $<0.001$ \\
\hline Counseling skill & 2.14 & 0.56 & 3.85 & $<0.001$ \\
\hline Medication knowledge & -0.35 & 0.65 & -0.54 & 0.29 \\
\hline Psychological sickness & 0.71 & 0.14 & 4.89 & $<0.001$ \\
\hline Prepaid & 0.056 & 0.23 & 0.24 & 0.41 \\
\hline Age & 2.89 & 0.82 & 3.54 & $<0.001$ \\
\hline Ethnicity & -0.03 & 0.23 & -0.15 & 0.44 \\
\hline Family practice & -0.40 & 0.27 & -1.48 & 0.07 \\
\hline \multicolumn{5}{|l|}{ Variance parameter } \\
\hline Counseling skill & 4.86 & 4.12 & 1.18 & 0.12 \\
\hline Medication knowledge & 3.21 & 1.65 & 1.95 & 0.03 \\
\hline
\end{tabular}




\begin{tabular}{|c|c|c|c|c|c|}
\hline & Counseling & Antidepressants & $\begin{array}{l}\text { Minor } \\
\text { tranquilizer }\end{array}$ & $\begin{array}{l}\text { No } \\
\text { treatment }\end{array}$ & $\begin{array}{l}\text { Change in } \\
\text { appropriate care } \\
\text { (counseling or } \\
\text { antidepressants) }\end{array}$ \\
\hline \multicolumn{6}{|c|}{ (i) Among 100 patients with double (severe) depression } \\
\hline $\begin{array}{l}\text { Shift all patients from fee for } \\
\text { service to prepaid }\end{array}$ & -10 & +6 & +3 & +1 & -4 \\
\hline $\begin{array}{l}\text { Increase medication knowledge by } \\
40 \text { points }\end{array}$ & +4 & +5 & -6 & -3 & +9 \\
\hline $\begin{array}{l}\text { Increase counseling skills by } 40 \\
\text { points }\end{array}$ & +3 & -3 & +15 & -15 & 0 \\
\hline $\begin{array}{l}\text { Shift all patients from fee-for- } \\
\text { service to prepaid care plus } \\
\text { increased medication knowledge }\end{array}$ & -7 & +11 & -3 & -1 & +4 \\
\hline \multicolumn{6}{|c|}{ (ii) Among 100 patients with subthreshold (minor) depression } \\
\hline $\begin{array}{l}\text { Shift all patients from fee for } \\
\text { service to prepaid }\end{array}$ & -8 & 0 & 0 & +8 & NA \\
\hline $\begin{array}{l}\text { Increase medication knowledge by } \\
40 \text { points }\end{array}$ & +3 & 0 & -1 & -2 & NA \\
\hline $\begin{array}{l}\text { Increase counseling skills by } 40 \\
\text { points }\end{array}$ & +2 & 0 & +5 & -7 & NA \\
\hline $\begin{array}{l}\text { Shift all patients from fee-for- } \\
\text { service to prepaid care plus } \\
\text { increased medication knowledge }\end{array}$ & -5 & 0 & 0 & +5 & NA \\
\hline
\end{tabular}

Note: We simulate the changes in knowledge using the MOS distribution for other variables (i.e. payment is about half fee for service, half prepaid); similarly, we simulate the changes in payment using the MOS distribution for other variables and knowledge.

appropriate treatment, defined for severe depression only as the sum of patients receiving counseling or effective dosages of antidepressants. It is unclear what appropriate care is for minor depression, so the last column does not apply for subthreshold patients.

We estimate that a switch from fee-for-service to prepaid care results in ten fewer severely depressed patients counseled out of 100, six more would use effective antidepressants and three more would receive minor tranquilizers, but there is little effect on the number without treatment and a modest decrease in the number of severely depressed patients with any appropriate care (four out of 100). There is no detectable effect of type of payment on targeting treatment to sicker patients; counseling rates drop by eight out of 100 minor depression patients and by ten out of 100 severely depressed patients. Put differently, 'turning back the clock' to unmanaged fee-for-service care would do little to change the overall percentage of patients treated, but would result in a shift toward counseling and away from medicationbased solutions, including both effective antidepressant medications and ineffective minor tranquilizers (if the relationships between knowledge and use of both of these medications that were observed during the MOS still applied today).

In contrast, we estimate that for sicker patients, increased antidepressant medication knowledge would raise the overall level of appropriate care for nine patients in 100, through higher rates of use of counseling and antidepressant medication, and use of regular minor tranquilizers falls for six more of 100 patients, while increased medication knowledge has little effect on care of minor depressed patients. Increased perceived counseling skills, controlling for medication knowledge, would markedly raise minor tranquilizer use (15 of 100 severely depressed patients) with no effect on the overall level of appropriate care. The pattern is similar at lower absolute levels for minor depression patients: five more of 100 patients use minor tranquilizers with greater perceived physician counseling skill. Because the medication knowledge and counseling skills scales are correlated 0.5 , the effect of increased counseling skill without controlling for medication knowledge is more similar in direction to that of improving medication knowledge, but the unique effect of perceived counseling skills seems to be to prefer minor tranquilizers over antidepressant medication, raising questions about what this measure means in a sample of primary care clinicians with little formal training in counseling; specifically, it may be a marker for relative aversion to antidepressant medication use, which in a primary care provider sample with few other treatment options could reflect a propensity to provide poor quality of care, rather than better counseling.

A simultaneous shift from fee-for-service to prepaid care plus improving antidepressant knowledge increases overall appropriate care for severe depression (by four of 100 patients) and shifts care away from counseling (seven of 100 patients) to antidepressant medication (increase of 11 
in 100 patients), while reducing use of minor tranquilizers somewhat (three more of 100 patients). This simulated strategy also reduces treatment to subthreshold patients, entirely through reducing counseling rates (for five out of 100 patients). In Table 4(i) the greatest increase in appropriate care for severely depressed patients occurs with better medication knowledge (nine of 100 patients), holding financing constant, but this strategy also slightly increases counseling of subthreshold patients (two more in 100 patients, Table 2(ii)).

While the empirical estimates and their statistical significance provide evidence in favor of the theory presented in this paper, the substantive impact on health and cost outcomes of changes in knowledge versus shifts in financing are not obvious from the regression model or the simulated changes in treatment. However, the most important dimensions for policy purposes are health outcomes and costs. To simulate the effects of payment switches or an information increase on treatment costs and morbidity outcomes for a more general population, we have to go beyond our estimated model and need three additional assumptions.

Assumption 1 (prevalence). In the primary care practices studied in the MOS, the prevalence of at least some depressive symptoms was about $25 \%$ and the prevalence of a current unipolar depressive disorder was about $7 \% .^{2}$ In a health plan that has 100000 primary care patients over a year (such as an HMO with 150000-200000 enrollees), 7000 patients would have a depressive disorder. Assumption 2 (processes of care-outcomes link). Counseling and antidepressant medication improve functioning among patients with a disorder, but have no effect on patients without a disorder. We use the effect sizes reported by Sturm and Wells. ${ }^{24}$

Assumption 3 (processes of care-health care cost links). We only calculate direct health care costs (not indirect or other social costs) and only those that are directly related to measured processes of care. This includes medication costs, additional visits typically associated with medication use or counseling sessions (see the article by Sturm and Wells ${ }^{24}$ for a detailed breakdown). Thus, the cost consequences are most meaningful for an HMO or insurer responsible for treatment costs.

Under these assumptions, we apply changes at the top of Table 4 to all patients with depressive disorder and the changes at the bottom of Table 4 to all subthreshold patients. Table 5 shows that the switch from fee-for-service to prepaid care for 100000 patients would probably save over one million dollars for depression care annually, but could lead to an additional 175 additional serious functional limitations (such as not being able to work at a paying job, work around the house or engage in moderate or vigorous physical activities) over care as usual, given the expected changes in process of care. In contrast, increased medication knowledge (in a given financing systems) raises costs by less than $\$ 600000$ and reduces an additional 380 serious functioning limitations from care as usual, given the expected process changes. Combining the two strategies of educational interventions and changes in financing can achieve both cost savings (about $\$ 650000)$ and health improvements (175 additional functional limitations removed). We did not simulate costs and health outcomes of changing counseling skill because of uncertainty over its interpretation in this data set, and because the associated large increase in minor tranquilizer use observed in the MOS data would lead to increased costs and unimproved or poorer functioning outcomes; estimating the policy implications of improved (and scientifically correct) counseling skills requires another data base. We assume that estimating consequences of lower knowledge would be of little interest, although some proposed changes in staffing of primary care (less reliance on physicians) could possibly lower knowledge level of providers.

\section{Discussion}

The main goal of this paper has been to integrate health services research questions, which concern information and quality of care, and economic questions, which concern incentives, into one model and to provide an empirical application of this approach. This paper has focused on physician behavior, in contrast to many models in health economics, which start with consumer demand. However, consumer demand models quickly become intractable when one tries to study provider knowledge, ${ }^{27}$ a reason why health economics has not had a strong influence in health services research that is concerned with incomplete information, which includes the large areas of quality of care, practice guidelines or provider education. By focusing on providers in an agency framework, this model circumvents the intractability of standard market models, but gives up some traditional economic questions, such as market equilibria, at least in this first model. Nevertheless, this approach could introduce economic models to large areas in health care that influence public policies, yet so far have remained outside the scope of economic research, and lead to a closer integration between medical and economic research questions. Such an integration would also help to improve the quality of data for economic analyses.

We used simulation to compare the likely effects of changing provider knowledge about depression treatment in primary care with the effects of a shift toward prepaid managed care as opposed to fee-for-service care. We distinguished increased preferences for appropriate treatment choices and improved targeting of appropriate treatments to sicker patients, and estimated the treatment costs and morbidity consequences of changes in quality of care. Our study supports several broad conclusions: increasing provider knowledge, if it is achievable, could have the highly desirable effect of greater targeting of treatments to sicker patients while not raising overall treatment rates much-a treatment pattern that many hoped managed care could achieve, but for which there has been little evidence. We estimated that a change in knowledge, if achieved in the current system, would also result in a moderate increase in 


\begin{tabular}{lcc}
\hline & Costs & $\begin{array}{c}\text { Improved health (number of } \\
\text { serious functional limitations } \\
\text { reduced) }\end{array}$ \\
\hline Shift all patients from fee for service to prepaid & $-\$ 1220000$ (reduction) & -175 (deterioration) \\
$\begin{array}{l}\text { Increase medication knowledge by 40 points } \\
\text { Shift all patients from fee-for-service to prepaid care plus increased } \\
\text { medication knowledge }\end{array}$ & $+\$ 580000$ (increase) & +380 (improvement) \\
\hline
\end{tabular}

Note: We simulate the changes in knowledge using the MOS distribution for other variables (i.e. payment is about half fee-for-service, half prepaid); similarly, we simulate the changes in payment using the MOS distribution for other variables and knowledge.

treatment costs, but substantially improved outcomes among more severely depressed patients.

The difference between prepaid managed care and feefor-service care, as these systems existed in the late 1980s, was associated with a different pattern of results. We found that prepayment led to a general shift away from counseling and toward medication management (psychotherapy and antidepressant medication are both of established efficacy), across sickness levels. That is, there is no greater targeting of sicker depressed patients in prepaid care. We estimated that the changes in process would result in a substantial cost savings, at the price of worse functioning outcomes. Conversely, a return to unmanaged fee-for-service care as it existed in the 1980s would be expected to somewhat increase overall rates and costs of care and shift care toward counseling (greater time spent in psychosocial management), unless trends in recent years and dissemination of SSRIs (introduced at the beginning of the MOS), has caused an irreversible shift toward medication management. Proponents of unmanaged care would probably have to implement disease management for depression care and other strategies derived from managed care to contain costs under a feefor-service system to remain financially viable, and this is probably one reason why such elements of managed care are in fact rapidly disseminating in fee-for-service systems of care.

Our analysis illustrates the value of considering these widely different policy goals simultaneously. We learned that variation in physician knowledge generally had stronger associations with clinically relevant practice patterns for depression than did a complete change in financing strategy. The moderate change in perceived knowledge we simulated (not near the extremes of observed values of perceived knowledge) was associated with enough improvement in appropriateness of care to more than offset the reduction in appropriateness with a complete shift from fee-for-service to prepaid managed care. Thus, joint consideration of policy alternatives can facilitate the identification of potential solutions to difficult clinical and policy problems, namely the reduction in quality of care for depressed patients under prepaid managed care, which is nevertheless rapidly growing in the US market. Of course, such a complete payment shift could cause changes in supply of physicians and other resources that could lead to greater changes in quality of care in the long run, such as less access to knowledgeable providers, but this is outside the scope of this paper or our data.

While there may be no perfect strategy to simultaneously improve quality of care and targeting of treatment to the sickest patients while containing health care costs and improving the cost-effectiveness of care, the strategy of combining prepaid care with greater physician knowledge could offer an advantage for plans and society. We estimated this combined strategy could lead to reduced costs of careabout half the savings we estimated for a shift to prepaid care without greater physician knowledge-and improved functioning outcomes-about half the improvement we estimated for greater physician knowledge without shifting to prepaid care.

One additional criterion that should be considered in evaluating alternative policies is social costs. Our calculations of costs only referred to direct treatment costs, which is most important to parties responsible for treatment costs, but policy decisions should also consider indirect social costs, such as improved productivity of better treated patients. Unfortunately, the effects of treatment on social costs remain speculative, which is why we focus on direct costs. The most reliable social cost estimate we can offer is that a decrease of one serious functional limitation increased family income by $\$ 2000$ or more over time over no such outcome improvement. ${ }^{24}$ Based on this estimate, the additional health improvements associated with increased medication knowledge in the hypothetical population in Table 5 (380 fewer functional limitations) would lead to increased family income of $\$ 760000$, thus more than offsetting the increased health care costs $(\$ 600000)$ and resulting in a net social gain financially. In this case, both direct treatment and societal costs are reduced, and, in the first year of outcome improvement alone, the combination of reduced treatment costs $(\$ 650000)$ and reduced social costs (increased family income of $\$ 350000$ ) could equal $\$ 1$ million over 100000 patients, which is likely to exceed the net social gains (after factoring in income losses from health deteriorations) of a full shift to prepaid care without changing provider knowledge. Of course, family income is not equivalent to all social costs and certainly not comprehensive, but it is a broad measure that can capture the improvements in productivity of the patient and family members associated with better health and even changes in the time costs of receiving care. 
A substantial limitation of this study is that information is not easy to measure and we ideally would like to rely on an objective test that scores each provider's knowledge against current scientific knowledge. Instead, our measures relied on self-report of knowledge and skill, which measures objective knowledge with error. For a simple analysis (bivariate regression), this error would underestimate the effect of objective information, but in the complex model we used, the direction of bias is unknown. Of course, the model provides unbiased estimates of the effects of perceived knowledge. Perceived knowledge or skill may reflect scientifically outdated or false knowledge, and the example of perceived counseling skill in this analysis could offer such a case because it was associated (controlling for medication knowledge) with greater use of minor tranquilizers. Nevertheless, this finding is interesting because it suggests that primary care providers who counsel may overestimate the benefit of regular use of minor tranquilizers, which are not efficacious in severe depression. This further suggests that training programs should provide education in appropriate medication management when teaching counseling skills. In contrast, our measure of antidepressant medication knowledge appears to be a valid competence measure, supported by evidence from a longitudinal analysis that improved knowledge predicts greater rates of maintenance therapy for recurrent depression over two years.

The reader may feel we have strayed far from the conventional experimental research paradigm in the clinical literature. Clearly, we rely on observational data and crosssectional variations, which makes any causal inferences (and we do interpret our model causally) less convincing than in an experimental trial. However, we have empirically estimated our model with actual data whereas studies of similar scope almost always have to rely on expert opinion or otherwise guess the central parameter. Many of the most relevant policy questions cannot be studied experimentally and our paper tries to bridge the large gap between experimental clinical research and research that is policy relevant.

\section{Acknowledgements}

We gratefully acknowledge financial support from NIMH grants MH54147 (Sturm) and MH54623 (Wells) and AHCPR grant HS08349. The full acknowledgments for the parent MOS investigators and practice administrators are provided in the article by Wells et al. ${ }^{1}$

\section{References}

1. Wells KB, Stewart A, Hays RD, Burnam MA, Rogers W, Daniels M, Berry S, Greenfield S, Ware J. The functioning and well-being of depressed patients: results of the medical outcomes study. J. Am. Med. Assoc. 1989; 262: 914-919.

2. Wells KB, Sturm R, Sherbourne CD, Meredith LS, Caring for Depression. Harvard University Press, Cambridge, CA, 1996.

3. Broadhead WE, Blazer DG, George LK, Tse CK. Depression, disability days, and days lost from work in a prospective epidemiologic survey. J. Am. Med. Assoc. 1990; 264: 2524-2528.

4. Greenberg PE, Stiglin LE, Finkelstein SN, Berndt ER. The Economic burden of depression in 1990. J. Clin. Psychiatry 1993; 54: 405-418.

5. Depression Guideline Panel. Depression in Primary Care Vol. 1. Detection and Diagnosis (Clinical Practice Guideline 5). AHCPR Publication 93-0550. Rockville, MD: US Department of Health and Human Services, Public Health Service, AHCPR, 1993a.

6. Depression Guideline Panel. Depression in Primary Care Vol. 2. Treatment of Major Depression (Clinical Practice Guideline 5). AHCPR Publication 93-0551. Rockville, MD: US Department of Health and Human Services, Public Health Service, AHCPR, 1993b.

7. Ellis RP and McGuire TG, Supply-side and demand-side cost sharing in health care, J. Econ. Perspectives 1993; 7: 135-151

8. Linn LS, Yager J, Screening of depression in relationship to subsequent patient and physician behavior, Med. Care, 1982; 20: 1233-1240

9. Magruder-Habib K, Zung WK, Feussner JR, Improving Physicians' Recognition and Treatment of Depression in General Medical Care, Med. Care, 1990; 28: 239-250.

10. Brody DS, Lerman CE, Wolfson HG, Caputo GC, Improvement in physicians' counseling of patients with mental health problems. Arch. Intern. Med. 1990; 150: 993-998.

11. Andersen SM, Harthorn BH, Changing the psychiatric knowledge of primary care physicians. Gen. Hospital Psychiatry 1990; 12: 177-190.

12. Katon W, Von Korff M, Lin M, Walker E, Simon GE, Bush T, Robinson P, Russo J, Collaborative Management to achieve treatment guidelines. J. Am. Med. Assoc. 1995; 273: 1026-1031.

13. Callahan CM, Hendrie HC, Dittus RS, Brater DC, Hui SL, Tierney WM. Improving treatment of late life depression in primary care: a randomized clinical trial. J. Am. Geriatr. Soc. 1994; 42: 839-846.

14. Rutz W, Carlsson P, Von Knorring L, Walinder J, Cost-benefit analysis of an educational program for general practitioners by the Swedish Committee for the Prevention and Treatment of Depression, Acta Psychiatr. Scand. 1992; 85: 457-464.

15. Katon W, Von Korff M, Lin E et al. Adequacy and duration of antidepressant treatment in primary care. Med. Care 1992; 30: 67-76.

16. Lurie N, Moscovice IS, Finch M, Christianson JB, Popkin MK, 1992 Does Capitation Affect the Health of the Chronically Mentally Ill? Results from a Randomized Trial, J. Am. Med. Assoc. 267: 3300-3304.

17. Rogers WH, Wells KB, Meredith LS, Sturm R, Burnam A. Course of Depression for Adult Outpatients Under Prepaid or Fee-for-Service Financing. Arch. Gen. Psychiatry 1993; 50: 517-526.

18. Rosen S, and P. Taubman (1979) Notes on Information and Unproductive Consumption. Mimeo, Department of Economics, University of Chicago.

19. Gertler P, Sturm R, Davidson B. Information and the Demand for Supplemental Medicare Insurance. NBER working paper 4700, 1994.

20. Daganzo, C. (1979) Multinomial Probit, New York: Academic.

21. Bunch, DS (1991) 'Estimability in the Multinomial Probit Model,' Transportation Res. 25, 1-12.

22. Keane, MP (1992) 'A Note on Identification in the Multinomia Probit Model,' J. Business Econ. Stat. 10: 193-200.

23. Tarlov A, Ware JE, Greenfield S, Nelson EC, Perrin E, Zubkoff M. The Medical Outcomes Study: an Application of Methods for Monitoring the Results of Medical Care. J. Am. Med. Assoc. 1989; 262: 925-930.

24. Sturm R, Wells KB, How can care for depression become more costeffective? J. Am. Med. Assoc. 1995; 273: 51-58.

25. Wells KB, Katon W, Rogers WH, Camp P. Use of minor tranquilizers and antidepressant medications by depressed outpatients: Results from the Medical Outcomes Study. Am. J. Psychiatry 1994; 151: 694-700.

26. Sturm R, Jackson C, Meredith LS, Yip W, Manning WG, Rogers WH, Wells KB, Utilization of Outpatient Mental Health Care Among Depressed Patients under Prepaid or Fee-for-Service Financing. Health Services Res. 30: 319-340, 1995.

27. Pauly, MV (1980) Doctors and Their Workshops. Chicago, IL: University of Chicago Press. 